\title{
Minimal walking on the lattice
}

\author{
Simon Catterall \\ Department of Physics, Syracuse University, Syracuse, New York 13244-1130, USA \\ Francesco Sannino \\ CERN Theory Division, CH-1211 Geneva 23, Switzerland \\ and University of Southern Denmark, Campusvej 55, DK-5230 Odense M, Denmark
}

(Received 13 May 2007; published 15 August 2007)

\begin{abstract}
We provide the first evidence of a walking dynamics for two color lattice Yang-Mills theory with two Dirac flavors in the symmetric representation of the gauge group.

DOI: 10.1103/PhysRevD.76.034504

PACS numbers: $11.15 . \mathrm{Ha}, 12.38 . \mathrm{Gc}, 12.60 . \mathrm{Nz}$
\end{abstract}

\section{INTRODUCTION}

We undertake the first numerical study of a four dimensional asymptotically free gauge theory with dynamical fermions transforming according to the two-index symmetric representation of the gauge group. To be more precise we consider the simplest of these theories with interesting dynamics and phenomenological applications, i.e. a two color gauge theory with two Dirac fermions transforming according to the two-index symmetric representation. For two colors the two-index symmetric coincides with the adjoint representation and the reality of the representation enhances the quantum flavor symmetry group to $S U(4)$. Remarkably this theory coincides with the fermionic sector of the $\mathcal{N}=4$ supersymmetric theory with two colors.

Recently it has been argued that this theory lies close to a nontrivial infrared fixed point [1]. In this case the coupling constant will run very slowly from the infrared to the ultraviolet; the coupling is said to walk. By analyzing the phase diagram as a function of the number of colors and flavors in $S U(N)$ gauge theories with Dirac fermions in a given arbitrary representation of the gauge group we have shown that this theory is minimal in the sense that it is the theory with the smallest number of flavors (above one) which exhibits such walking dynamics [2].

The walking dynamics is expected to be dramatically different than in the QCD case. This is so since the presence of a nearby IR fixed point should generate an anomalously small mass scale and ensure that long distance quantities are insensitive to the short distance coupling. The physics of the fixed point theory per se is very interesting and when coupled to nonconformal theories (such as the standard model) in the way described recently by Georgi [3] it leads to interesting experimental signatures. This is so since the presence of a conformal symmetry signals itself experimentally in a way that formally resembles the production of a noninteger number of massless invisible particles. The noninteger number is nothing but the scale dimension of the operator, coming from the underlying conformal theory, coupled weakly to the standard model operators. However, as is also stressed by Georgi, very little is known about conformal or nearconformal theories in four dimensions because of the complicated nonperturbative dynamics. Our work should be considered as a first step in this direction.

In addition, the emergence of a walking property for just two Dirac flavors renders this theory an ideal candidate to break the electroweak theory dynamically [1]. This extension of the standard model passes the stringent electroweak precision constraints [4]. Moreover, it allows for a successful unification of the standard model couplings [5] and allows us to construct different types of dark matter candidates [6-8].

The lattice results presented in this work support the theoretical expectations, i.e. that the present theory walks. To arrive to this conclusion we analyze various physical quantities on the lattice and then compare them to the ones for a two color gauge theory with two Dirac flavors in the fundamental representation of the gauge group. The latter theory, as is clear from the phase diagram reviewed later, is very far away from an infrared fixed point. Although our simulations employ dynamical quarks the lattices we use are small so these results should only be taken as indicative of qualitative agreement with the theoretical expectations and encourage one to embark on a more serious study on larger lattices.

In the next section we review the expected phase diagram as a function of flavors and colors for nonsupersymmetric asymptotically free $S U(N)$ gauge theories with $N_{f}$ Dirac fermions in a given representation of the gauge group [2]. Here it is shown that the model we study in this paper is indeed the theory with the lowest number of Dirac flavors (above one) able to feature walking. This feature makes this theory also an ideal candidate when used for breaking the electroweak theory dynamically. We review the salient features and the notation here.

We then describe the details of our lattice theory and simulation algorithm. This is followed by a detailed description of our numerical results. We compare them to the theory with fermions in the fundamental representation. Our results show clear differences between the two theories - the symmetric quark theory has a behavior similar to the fundamental quark theory at strong coupling but deviates substantially for weak coupling where we observe substantially lighter hadronic masses. 


\section{REVIEW OF SOME THEORETICAL AND PHENOMENOLOGICAL ASPECTS OF HIGHER DIMENSIONAL REPRESENTATIONS}

The phase diagram of strongly coupled theories is relevant both theoretically and phenomenologically. By comparing the dynamics of various strongly coupled theories in different regimes we acquire a deeper understanding of nonperturbative dynamics.

\section{A. Conformal window}

Recently we have completed the analysis of the phase diagram of asymptotically free nonsupersymmetric gauge theories with at least two Dirac fermions in a given arbitrary representation of the gauge group as a function of the number of flavors and colors [2]. With the exceptions of a few isolated higher dimensional representations below nine colors (fully investigated in [2]) the main phase diagram taken from [2] is sketched in Fig. 1. The analysis exhausts the phase diagram for gauge theories with Dirac fermions in arbitrary representations and it is based on the ladder approximation presented in $[9,10]$. Further studies of the conformal window and its properties can be found in [11-13].

In the plot the shaded areas represent the conformal windows for the fundamental representation, two-index antisymmetric, two-index symmetric, and adjoint representation. For each representation the upper solid curve

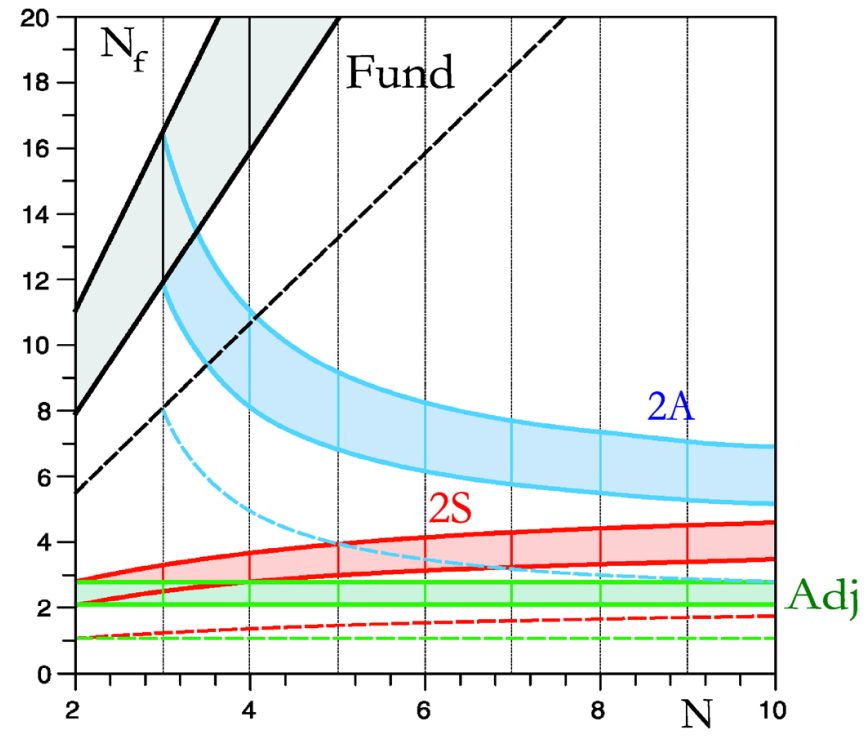

FIG. 1 (color online). Phase diagram for theories with fermions in the: (i) fundamental representation, (ii) two-index antisymmetric, (iii) two-index symmetric, (iv) adjoint representation as a function of the number of flavors and the number of colors. The shaded areas depict the corresponding conformal windows. The upper solid curve represents the loss of asymptotic freedom, the lower curve the loss of chiral symmetry breaking. The dashed curves show the existence of a Banks-Zaks fixed point. Picture taken from [2]. represents the loss of asymptotic freedom, the lower curve the loss of chiral symmetry breaking. The dashed curves show the existence of a Banks-Zaks fixed point [14]. Note how consistently the various representations merge into each other when, for a specific value of $N$, they are actually the same representation.

Remarkably the adjoint and the two-index symmetric representation need a very low number of flavors, for an arbitrary number of colors, to be near an infrared fixed point. For any number of colors, in the case of the adjoint representation and for two colors in the case of the symmetric representation the estimated critical lower number of flavors above which the theory is already inside the conformal window and hence no chiral symmetry breaking occurs is $N_{f}^{c} \sim 2.075$ [1,2]. Since this theory walks already for two flavors and hence has also a nontrivial chiral dynamics it will be denoted the minimal walking theory.

The theoretical estimates of the conformal window presented above need to be tested further. The very low number of flavors needed to reach the conformal window makes the minimal walking theories amenable to lattice investigations.

We also note that the theory with two colors and two Dirac flavors in the fundamental representation is very much below the critical number of flavors needed to develop a nontrivial infrared fixed point and hence it features QCD-like dynamics.

\section{B. Minimal walking technicolor}

New strongly interacting theories can emerge in extensions of the standard model. For example, to avoid unnaturally large quantum corrections to the mass squared of the elementary Higgs one can replace it by a new strongly coupled fermionic sector. This is the technicolor mechanism [15]. The generation of the masses of the standard model fermions requires extended technicolor interactions. To avoid large flavor-changing neutral currents technicolor theories possessing a sufficient amount of walking [16-20] are needed. The simplest of such models which also passes the electroweak precision tests (like, for example, the experimental bounds on the oblique parameters) has fermions in higher dimensional representations of the technicolor gauge group [1,4]. We have shown in [2] that the minimal walking theory is also the minimal walking technicolor theory. Such a theory has also a number of desirable features. For example, together with a minimal modification of the SM fermionic matter content it yields a high degree of unification, at the one loop level, of the SM couplings [5]. Straightforward extensions of the minimal walking theory able to accommodate extended technicolor interactions can be constructed [21].

\section{LATTICE THEORY}

The lattice action we employ consists of the usual Wilson plaquette term 


$$
\begin{aligned}
S_{G}= & -\frac{\beta}{2} \sum_{x} \sum_{\mu>\nu} \operatorname{Re} \operatorname{Tr}\left(U_{\mu}(x) U_{\nu}(x+\mu) U_{\mu}^{\dagger}(x+\nu)\right. \\
& \left.\times U_{\nu}^{\dagger}(x)\right),
\end{aligned}
$$

together with the Wilson action for two Dirac quarks in the symmetric representation

$$
\begin{gathered}
S_{F}=-\frac{1}{2} \sum_{x} \sum_{\mu} \bar{\psi}(x)\left(V_{\mu}(x)\left(I-\gamma_{\mu}\right) \psi(x+\mu)\right. \\
\left.+V_{\mu}^{\dagger}(x-\mu)\left(I+\gamma_{\mu}\right) \psi(x-\mu)\right) \\
+\sum_{x}(m+4) \sum_{x} \bar{\psi}(x) \psi(x),
\end{gathered}
$$

where the symmetric links are given by

$$
V_{\mu}^{a b}(x)=\operatorname{Tr}\left(S^{a} U_{\mu}(x) S^{b} U_{\mu}^{T}(x)\right),
$$

and the matrices $S^{a}, a=1,2,3$ are a basis for the symmetric representation

$$
\begin{gathered}
S^{1}=\frac{1}{\sqrt{2}}\left(\begin{array}{ll}
1 & 0 \\
0 & 1
\end{array}\right), \quad S^{2}=\frac{1}{\sqrt{2}}\left(\begin{array}{ll}
0 & 1 \\
1 & 0
\end{array}\right), \\
S^{3}=\frac{1}{\sqrt{2}}\left(\begin{array}{cc}
1 & 0 \\
0 & -1
\end{array}\right) .
\end{gathered}
$$

We have simulated this theory over a range of gauge couplings $\beta=1.5-3.0$ and bare quark masses $m$ ranging from $-1.0<m<1.0$ on $4^{3} \times 8$ lattices using the usual hybrid Monte Carlo (HMC) algorithm [22]. We have focused on determining the critical line $m_{c}(\beta)$ needed to approach the continuum limit and a variety of meson masses and decay constants. Typically we generate between 400-2000 $\tau=1$ HMC trajectories. Periodic boundary conditions were used for all fields.

In tandem with these symmetric quark runs we have also simulated the theory with fundamental quarks. This allows us to make comparisons at identical lattice volumes and comparable couplings and masses and helps highlight the essential differences associated with the walking dynamics.

\section{NUMERICAL RESULTS}

\section{A. Pion and rho mass}

We estimate the hadron masses by suitable fits to corresponding time sliced averaged correlation functions

$$
G_{O}(t)=\sum_{x, y}\left\langle\bar{\psi}(x, t) \Gamma_{O} \psi(x, t) \bar{\psi}(y, 0) \Gamma_{O} \psi(y, 0)\right\rangle,
$$

where $\Gamma_{O}=\gamma_{5}$ for the pion and $\Gamma_{O}=\gamma_{\mu}, \mu=1,2,3$ for the rho (the latter being averaged over spatial directions $\mu$ ). In practice we use point sources located at (odd, odd, odd) lattice sites on the $t=0$ time slice. Since our lattices are so small we have simply thrown out the $t=0$ data point and fitted the remaining correlator to a simple hyper- bolic cosine of the form $a_{O} \cosh \left(m_{O}(t-L / 2)\right)$ to estimate the corresponding meson mass $m_{O}$.

Consider first the usual case corresponding to taking the quarks to lie in the fundamental representation of the gauge group. Figure 2 shows curves of the pion mass squared (lattice units) for fundamental quarks at several values of $\beta$ as a function of the bare quark mass $m=M a$. At each coupling $\beta$ we observe the usual linear variation of $m_{\pi}^{2}$ with bare quark mass $m$. We see that the pion mass attains a minimum value for some critical quark mass $m_{c}(\beta)$ which moves towards $m=0$ as $\beta$ increases. This is similar to the situation in QCD. Notice that the minimal pion mass increases with $\beta$ as a result of finite volume effects.

Contrast this behavior with the analogous curves for the symmetric representation in Fig. 3 for the same bare couplings $\beta$ and over the same range of bare quark mass $m$. Again, a Goldstone behavior is seen over some range of bare quark mass with a critical quark mass $m_{c}(\beta)$ that runs towards the origin as $\beta$ increases. Notice though that this linear regime appears distorted at larger $\beta$ which we interpret to be the result of strong finite volume effects. This is consistent with the small values for the pion mass $m_{\pi} \sim 0.5$ observed there. Indeed the minimal pion mass is a factor of 3-4 times smaller for the symmetric pions than pions built from fundamental quarks. The appearance of this light scale is the first indication that the dynamics of this theory could be quite different from QCD.

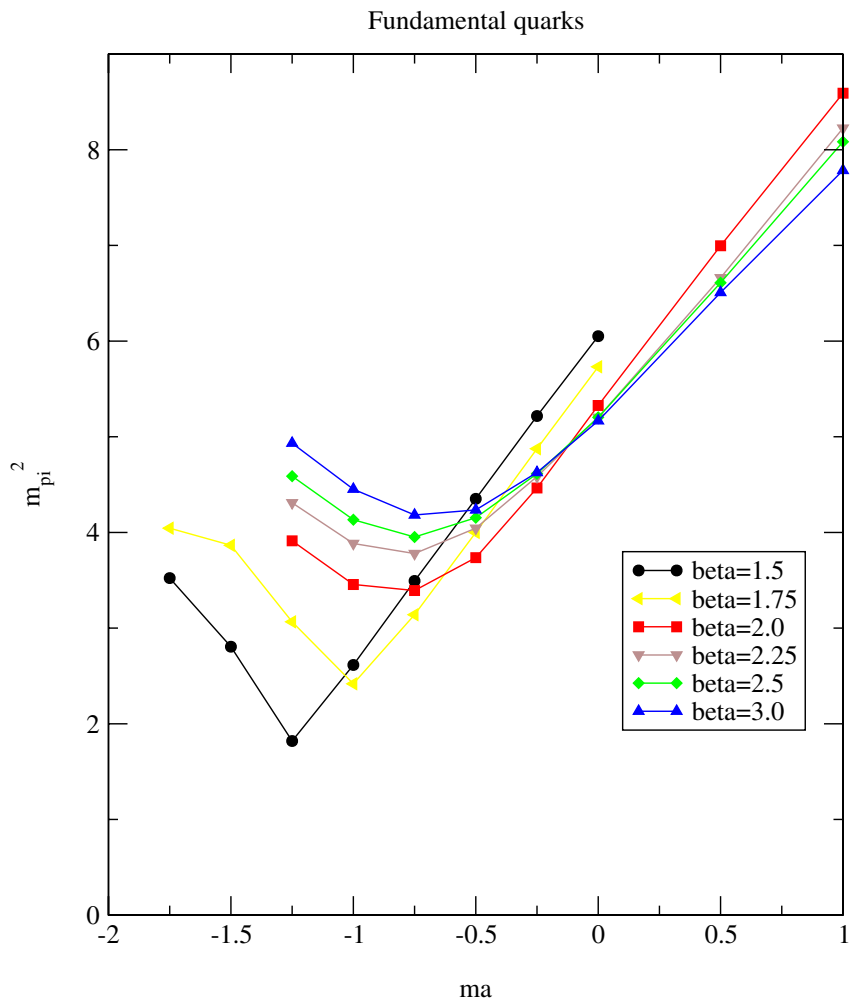

FIG. 2 (color online). Pion mass squared vs quark mass for fundamental quarks. 


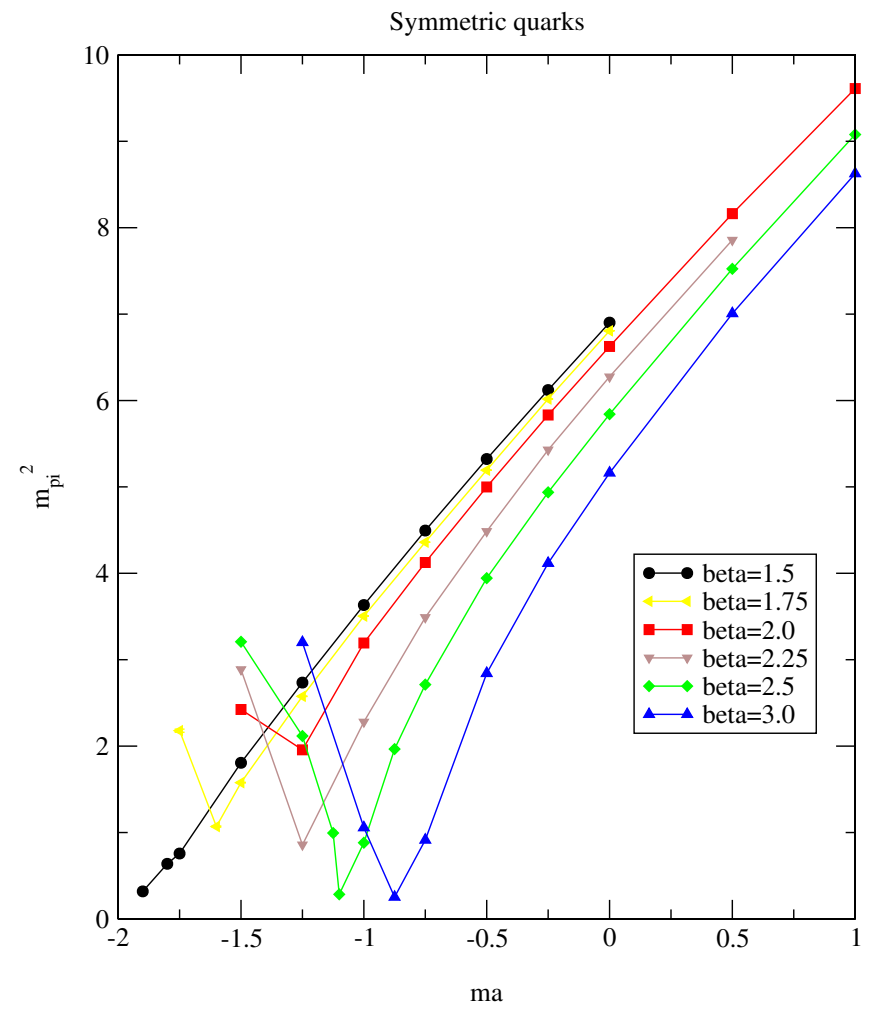

FIG. 3 (color online). Pion mass squared vs quark mass for symmetric quarks.
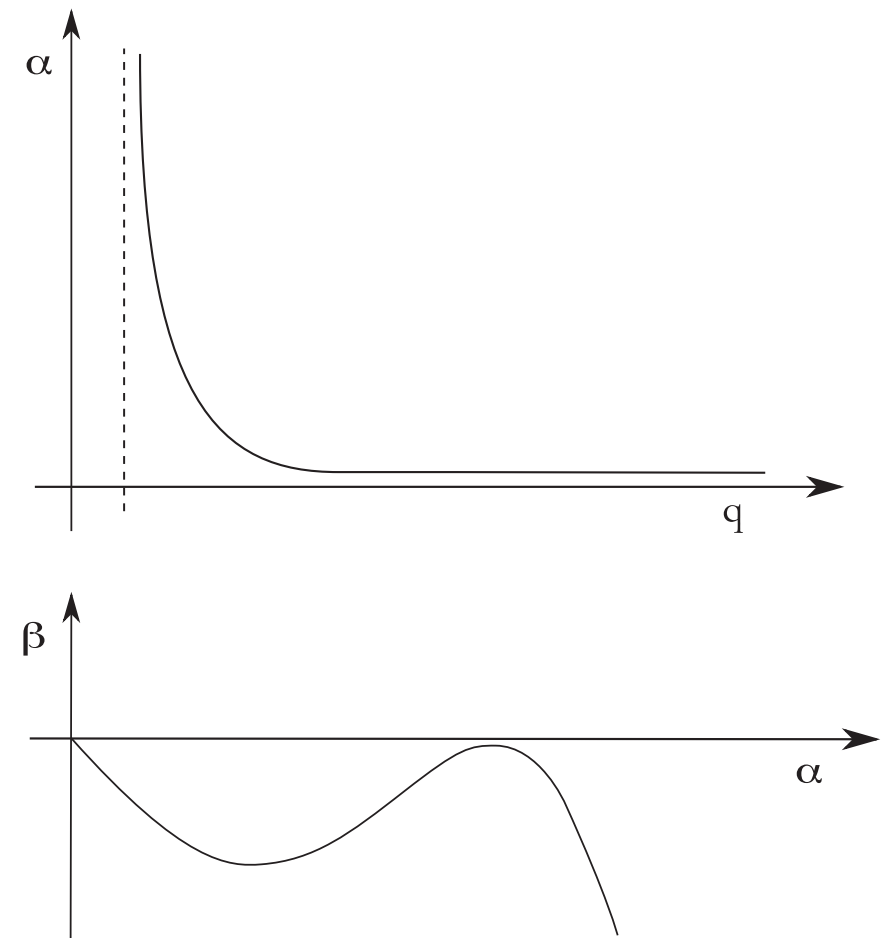

To make this clearer notice that the meson mass in lattice units is given in general by a pure number times a nonperturbative lattice scale parameter $\Lambda_{\text {latt }}$ which is related to the beta-function in the usual way

$$
\Lambda_{\text {latt }}=\Lambda_{\text {phys }} a \sim e^{-\int(d g) /(\beta(g))}
$$

This scale is small if the theory lies close to a zero of the beta-function. Furthermore, finite size effects are governed by the quantity $\Lambda_{\text {latt }} L$. For fixed $L$ the theory with the smaller $\Lambda_{\text {latt }}$ might be expected to exhibit larger finite size effects-as we observe for the symmetric quark theory.

However, perhaps the most striking difference between these symmetric plots and their fundamental cousins is the nonmonomtonic behavior of the pion mass with increasing $\beta$. For small $\beta$ the minimal pion mass increases with $\beta$ in a way which is similar to the fundamentals. However for $\beta>2$ the pion mass decreases with further increases in $\beta$. This is consistent with the existence of a beta-function (at zero quark mass) which resembles that of QCD at strong coupling but is small for couplings less than some characteristic value. Such a beta-function corresponds to the case of walking dynamics. Figure 4 gives a schematic picture of the beta-function and related coupling constant evolution for such a walking theory.

These differences are also seen in the rho meson. Figures 5 and 6 show the rho mass (in lattice units) as a

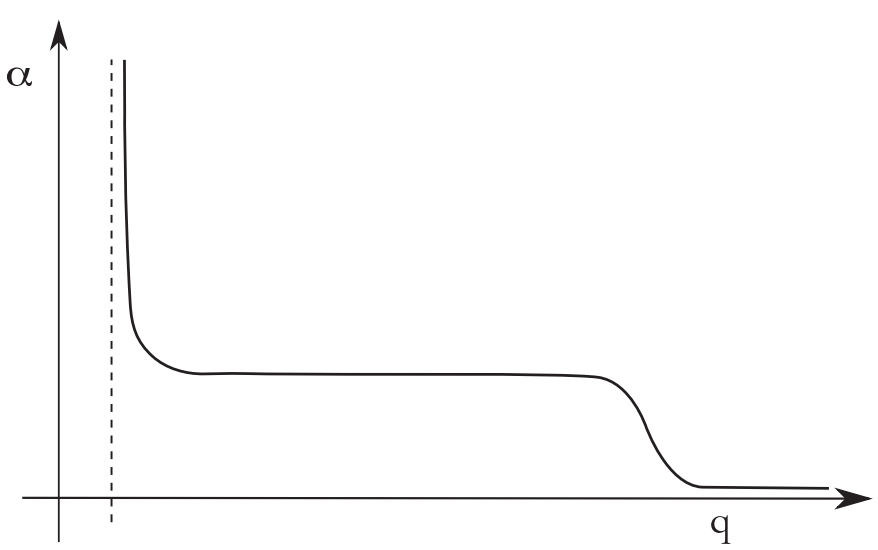

FIG. 4. Top-left panel: QCD-like behavior of the coupling constant as a function of the momentum (running). Top-right panel: Walking-like behavior of the coupling constant as a function of the momentum (walking). Bottom-right panel: Cartoon of the betafunction associated to a generic walking theory. 


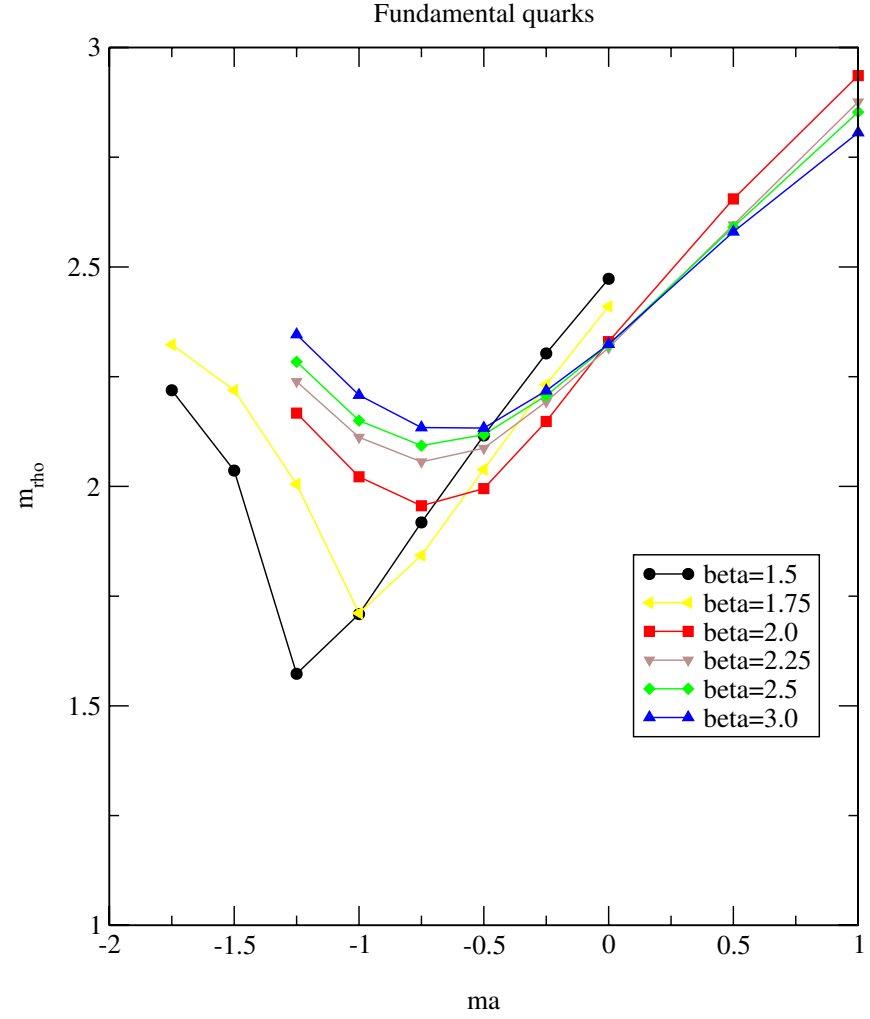

FIG. 5 (color online). Rho mass vs quark mass for fundamental quarks.

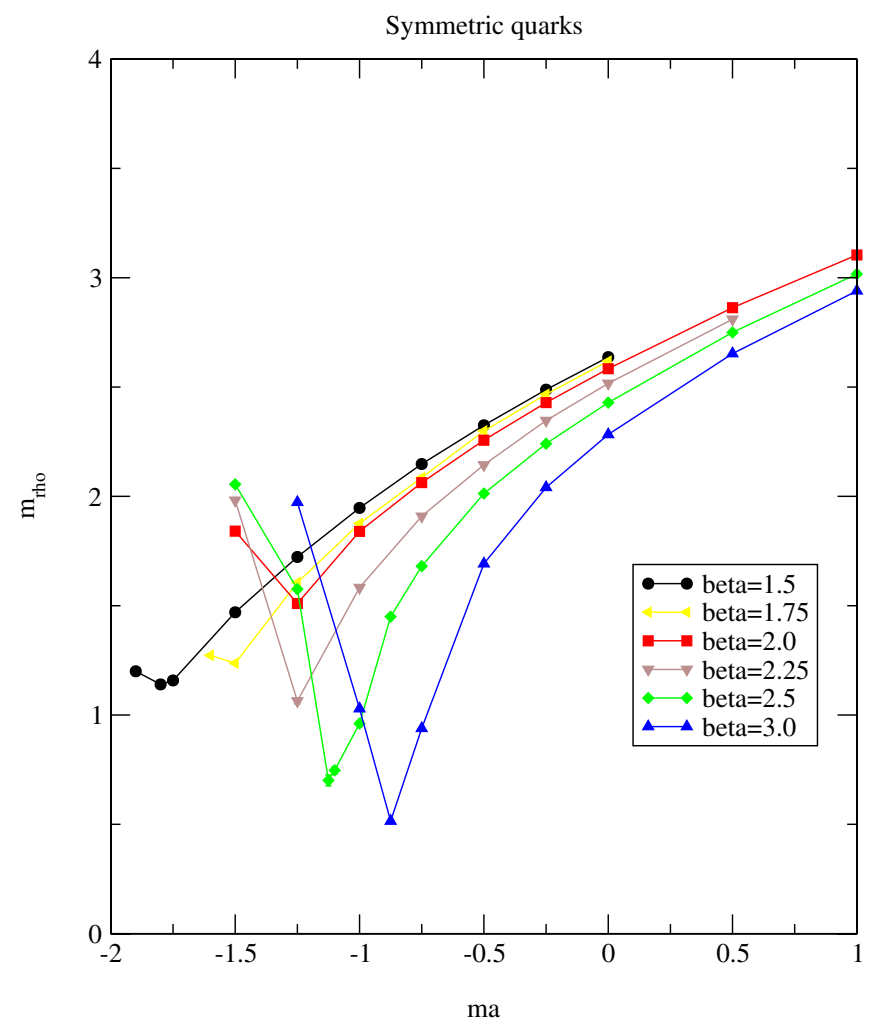

FIG. 6 (color online). Rho mass vs quark mass for symmetric quarks. function of quark mass over the same range of couplings $\beta$ for both fundamental and symmetric quarks. Again a minimal rho mass is seen which coincides approximately with the minimal pion mass and moves towards the origin as $\beta$ increases. In our later analysis we have used the minimal rho mass rather than that of the pion when trying to get an estimate of the critical line. In the case of fundamental quarks the observed rho mass increases monotonically with $\beta$ but again for symmetrics the minimal rho mass shows a nonmonotonic behavior with $m_{\rho}$ initially increasing and then falling as $\beta$ increases. The similar behavior of the rho mass to the pion mass for symmetric quarks lends strong support to the walking hypothesis since the nearconformal dynamics will suppress the mass of all hadron states - not just the pi meson.

\section{B. Pion decay constant}

To compute the pion decay constant we have also measured the axial correlator defined by

$$
G_{A}(t)=\sum_{x, y}\left\langle\bar{\psi}(x, t) \Gamma_{0} \Gamma_{5} \psi(x, t) \bar{\psi}(y, 0) \Gamma_{5} \psi(y, 0)\right\rangle .
$$

As for the hadron correlators we discard the $t=0$ data point and fit the remaining function to a hyperbolic sine function $a_{A} \sinh \left(m_{\pi}(t-L / 2)\right)$. We observe that the pion mass extracted from this fit is indeed consistent with that derived from the pion correlator in the physical regime

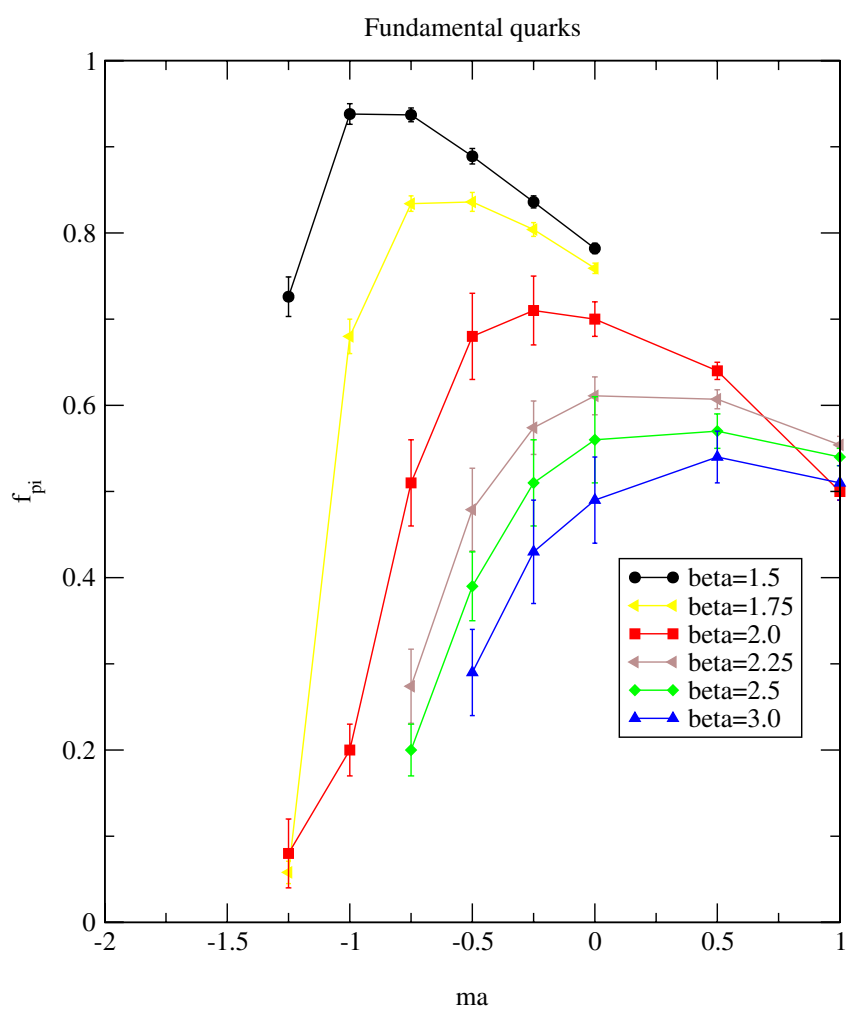

FIG. 7 (color online). $f_{\pi}$ vs quark mass for fundamental quarks. 


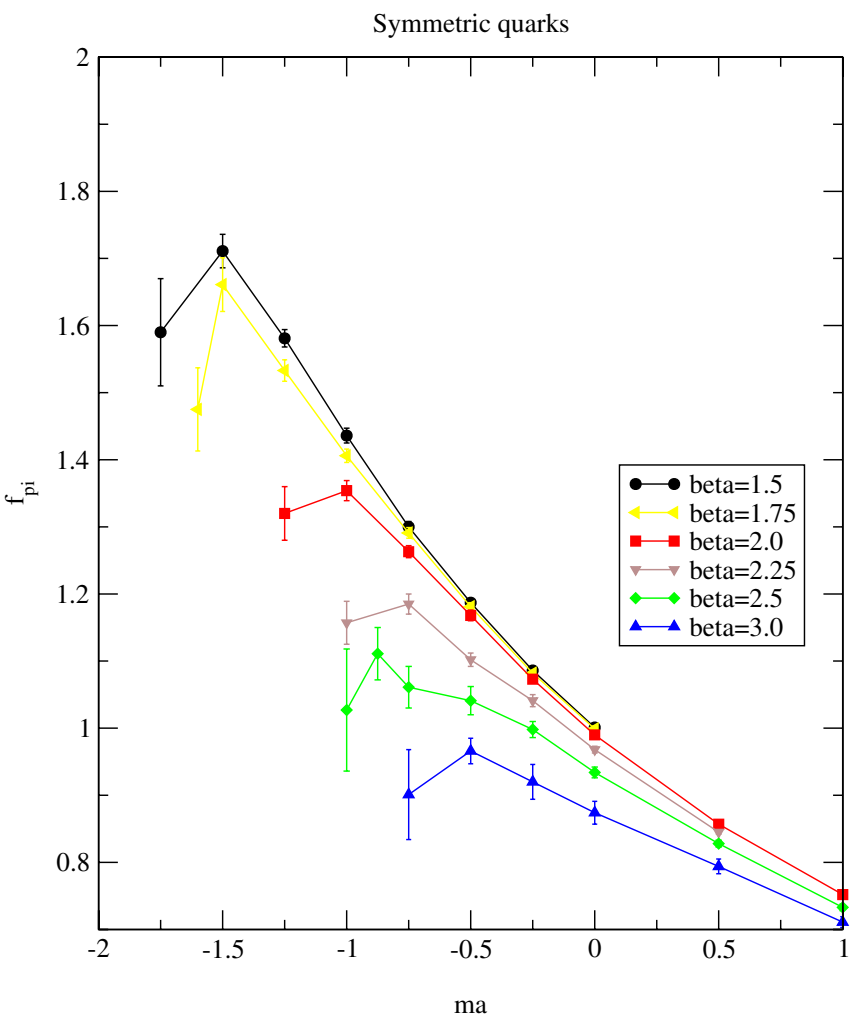

FIG. 8 (color online). $f_{\pi}$ vs quark mass for symmetric quarks.

$m>m_{c}(\beta)$. An expression for pion decay constant (in lattice units) is then given by [23]

$$
\frac{f_{\pi}}{Z}=\frac{a_{A}}{\sqrt{a_{\pi} m_{\pi}}} e^{m_{\pi} L / 4}
$$

We do not attempt in this paper to determine the renormalization constant $Z$ and hence all results for $f_{\pi}$ are only derived up this factor. Figure 7 shows this quantity for a range of $\beta$ and quark masses $m \geq m_{c}(\beta)$ for the theory with quarks in the fundamental. We see that $f_{\pi}$ increases as the critical line is approached with $f_{\pi}$ taking on a maximal value there before falling rapidly to zero in the phase with $m<m_{c}(\beta)$. The value of $f_{\pi}$ along the critical line falls as $\beta$ is increased and the lattice spacing reduced. Equivalent data for symmetric quarks is shown in Fig. 8. Notice that the maximal value of $f_{\pi}$ is about twice that found for fundamentals (where both are measured in lattice units) but otherwise the picture is qualitatively similar. Presumably the fact that $f_{\pi}$ is larger rather than smaller for symmetric quarks as compared to fundamentals, contrary to naive expectations, could be related to the differing renormalization constants $Z$ in the two cases.

\section{Scaling}

To examine the continuum limit in greater detail it is useful to examine dimensionless quantities as we scan in $\beta$ and $m$. Thus we have examined the ratio $\frac{f_{\pi}}{m_{\rho}}$ which is plotted in Figs. 9 and 10 over the same range of couplings and

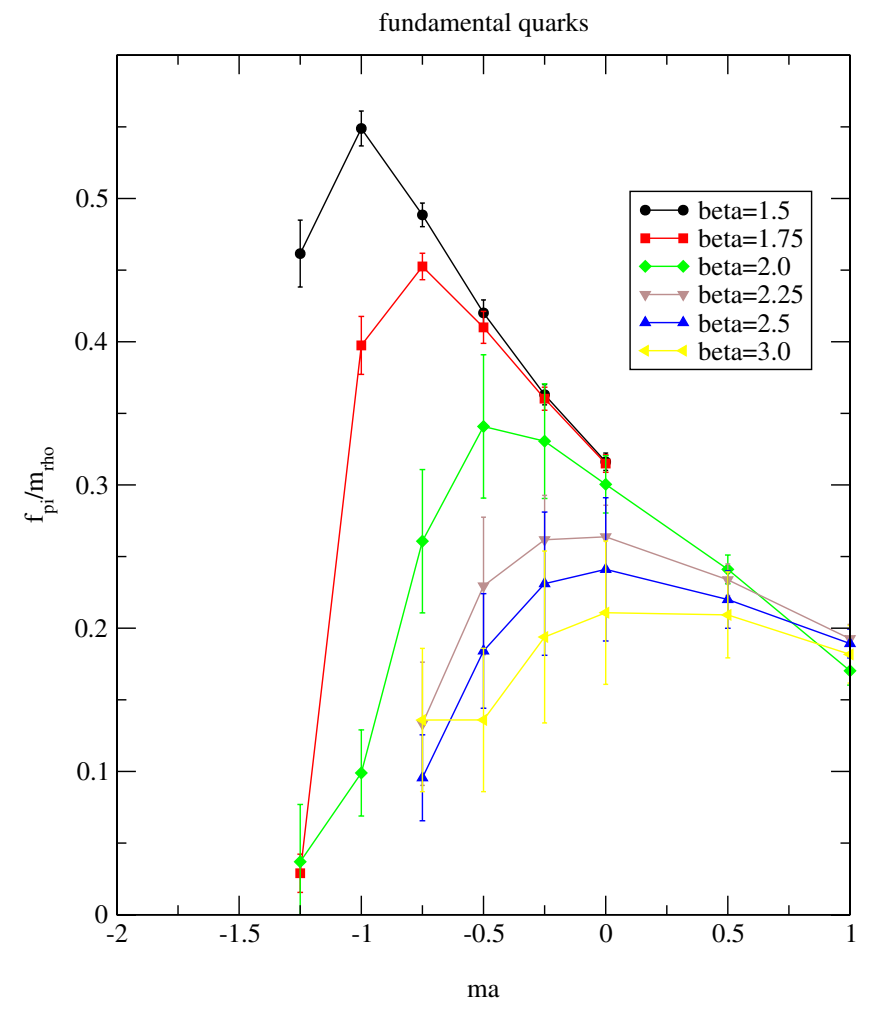

FIG. 9 (color online). $\frac{f_{\pi}}{m_{\rho}}$ vs quark mass for fundamental quarks.

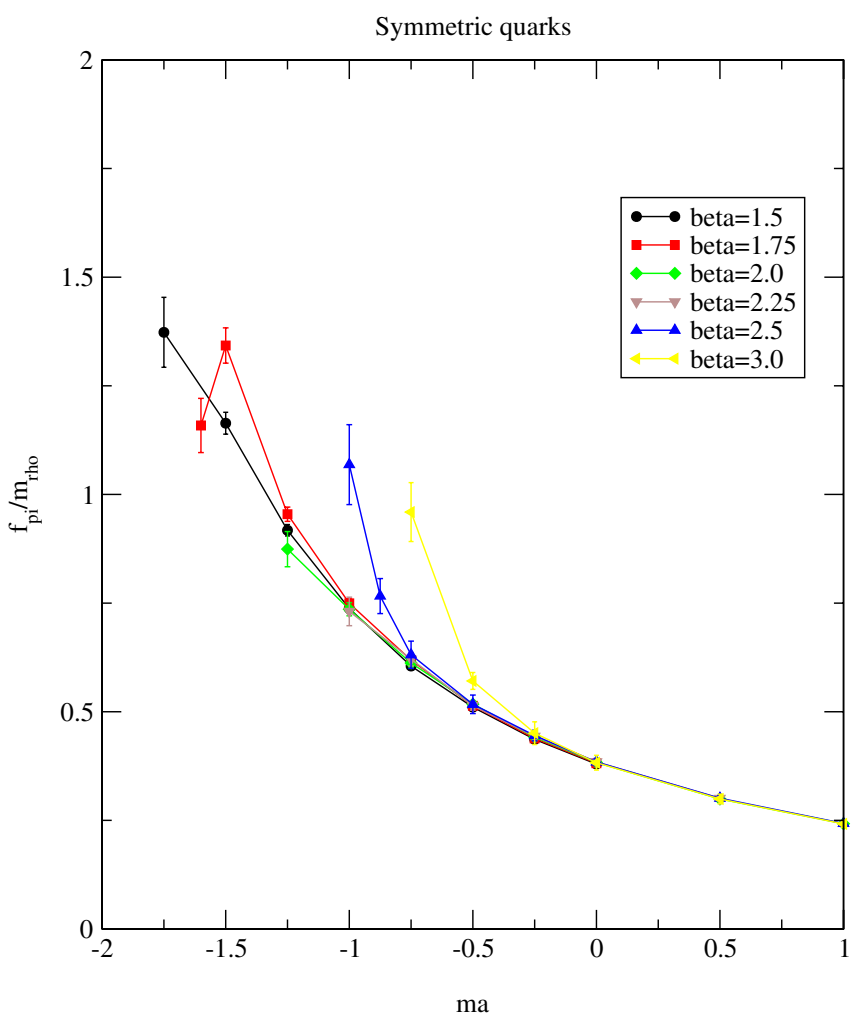

FIG. 10 (color online). $\quad \frac{f_{\pi}}{m_{\rho}}$ vs quark mass for symmetric quarks. 
masses. For both types of representations we see that $\frac{f_{\pi}}{m_{\rho}}$ increases as the quark mass is tuned towards the critical line. Furthermore there is evidence that for large enough quark mass the curves for different bare coupling $\beta$ lie on top of one another-this was not true for the bare $f_{\pi}$. Notice also that the values of $\frac{f_{\pi}}{m_{\rho}}$ for the two representations are much closer in this region of parameter space.

To extract continuum physics we should look for scaling-dimensionless quantities should become independent of coupling along the critical line as $\beta \rightarrow \infty$. We see some evidence for this away from the critical line where the sets of curves for different bare coupling $\beta$ lie on top of one another. This approximate collapse of the data onto a single scaling curve at intermediate quark masses happens for both representations of dynamical quark.

However, if we look at the values of $\frac{f_{\pi}}{m_{\rho}}$ for fundamental quarks near the critical quark mass $m \sim m_{c}$ we see that they decrease with increasing $\beta$. This is to be attributed to finite volume effects - as the lattice spacing decreases the physical volume also decreases since we use a fixed lattice volume. The rising rho mass with physical volume then leads to a falling value for this ratio. Notice also that the $\frac{f_{\pi}}{m_{\rho}}$ falls below the scaling curve as $m \rightarrow m_{c}$. This should be contrasted with the case of the symmetric quarks where the situation is reversed-the value of the ratio $\frac{f_{\pi}}{m_{\rho}}$ increases

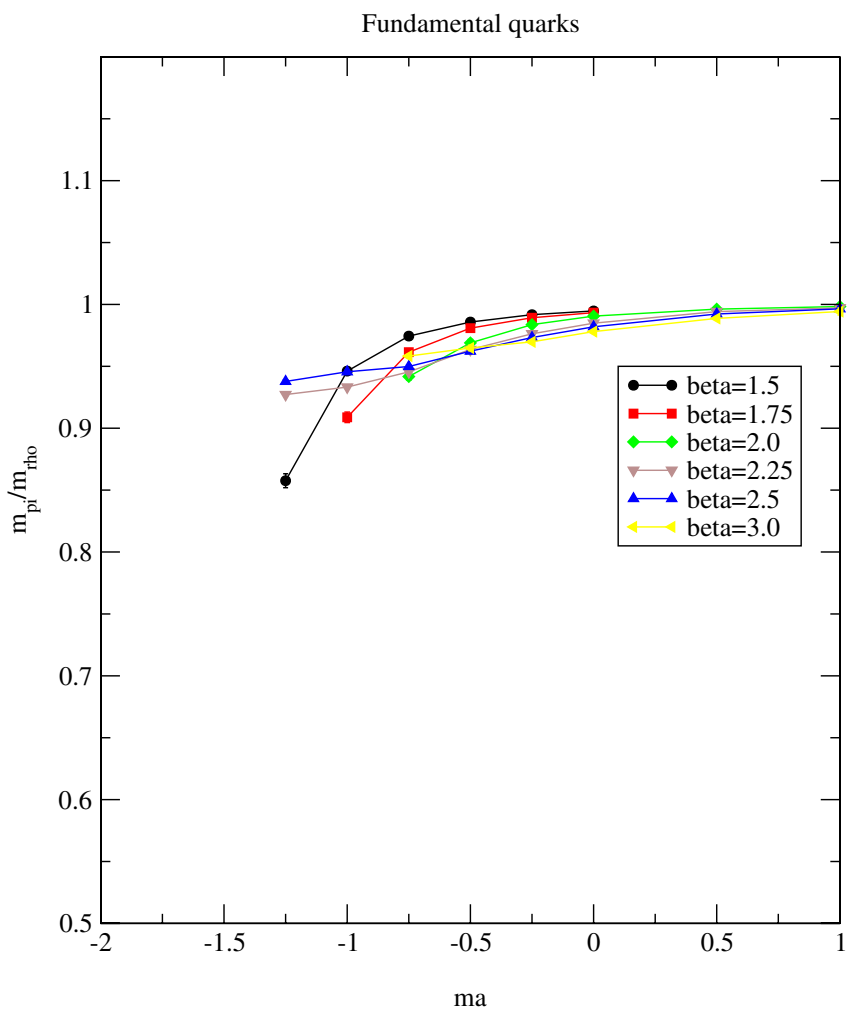

FIG. 11 (color online). $\frac{m_{\pi}}{m_{\rho}}$ vs quark mass for fundamental quarks.

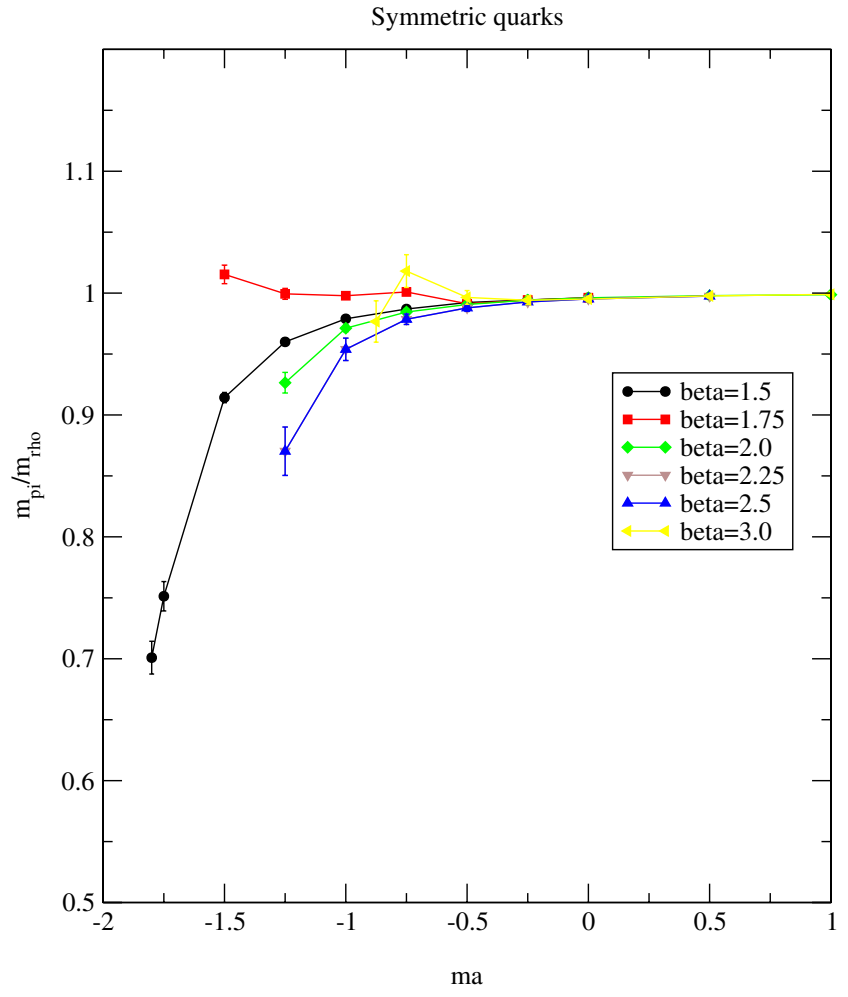

FIG. 12 (color online). $\quad \frac{m_{\pi}}{m_{\rho}}$ vs quark mass for symmetric quarks.

above the scaling curve as the quark mass is tuned towards its critical value.

Figures 11 and 12 show plots of the dimensionless ratio of pion to rho mass for the two theories. Both show values which decrease from unity as the critical line is approached. There is some evidence that the theory with symmetric quarks exhibits a value of the ratio closer to unity as the critical line is approached and that this limiting value is rather insensitive to the bare coupling for large $\beta$-a hint perhaps of walking dynamics.

\section{SUMMARY}

In this paper we report on the results of lattice simulations of two color QCD coupled to two flavors of quarks in the symmetric representation of the gauge group. These studies are motivated by the idea that this theory lies close to a strongly coupled conformal field theory associated to a new IR attractive zero of the beta-function. The resultant theory is expected to exhibit non-QCD-like dynamics and a slow evolution of the coupling constant over some range of scales.

To search for these effects we have simulated both the symmetric quark model and its counterpart employing fundamental quarks on identical lattice volumes and for comparable couplings and quark masses. In our simulations we observe several features which distinguish the symmetric from the fundamental representation. The most obvious of these can be seen in the hadron masses. 
While the symmetric pion and rho behave in a qualitatively similar way to their fundamental counterparts at strong coupling they depart from this behavior for $\beta \gtrsim 2.0$. In this regime their (lattice) masses decrease with further increases in the bare coupling $\beta$ attaining values substantially smaller than those seen in the theory with fundamental quarks. This observation is consistent with the existence of a light scale $\Lambda_{\text {latt }}$ attributable to walking dynamics.

However, our results can also be interpreted as evidence that for sufficiently light symmetric quarks and large $\beta$ the two color theory has already entered a conformal phase in which the quarks are deconfined at zero temperature. This could explain the lack of linear scaling of $m_{\pi}^{2}$ with $m$ at large $\beta$ since in such a phase chiral symmetry would be restored. If so, it is unclear whether this is the result of strong finite volume effects and will disappear on larger lattices or is a genuine bulk transition occurring for some $\beta_{c}$. If the latter scenario turns out to be true it would imply that the critical number of flavors needed to access the conformal phase is smaller than the perturbative estimate $N_{f}^{c}=2.075$. Further work on larger lattices will be needed to answer this question definitively.

In the case of fundamental quarks a much larger number of flavors is needed to approach the conformal window. Nevertheless, a small suppression of chiral symmetry breaking effects was observed in [24]. More recently a study of QCD with fundamental quarks showed evidence for an intermediate conformal phase for $7<N_{f}<17$ [25].
Exploratory simulations of this model with staggered quarks at finite temperature were conducted earlier [26]. Our results at zero temperature complement and extend that work.

Clearly our lattices are very small and so our results should be seen primarily as providing motivation for a study on larger lattices. Going to larger lattices will allow us to understand better the finite volume effects clearly visible in this work, will allow for a more careful study of the hadron spectrum, and should allow simulations to be undertaken at smaller cutoff. With a larger lattice it should also be possible to see direct evidence for walking by measuring the running of a renormalized coupling extracted from either the static quark potential or using Schrödinger functional techniques. This work is currently underway.

\section{ACKNOWLEDGMENTS}

We are happy to thank L. Del Debbio, M. T. Frandsen, and L. Giusti for helpful discussions. The work of S. C. is supported in part by DOE Grant No. DE-FG0285ER40237 while F. S. is supported by the Marie Curie Excellence Grant under Contract No. MEXT-CT-2004013510 as well as the Danish Research Agency. The numerical work was carried out using USQCD resources at Fermilab.
[1] F. Sannino and K. Tuominen, Phys. Rev. D 71, 051901 (2005).

[2] D. D. Dietrich and F. Sannino, Phys. Rev. D 75, 085018 (2007).

[3] H. Georgi, Phys. Rev. Lett. 98, 221601 (2007).

[4] D. D. Dietrich, F. Sannino, and K. Tuominen, Phys. Rev. D 72, 055001 (2005); 73, 037701 (2006). Note that we have also checked that our results do not change when taking into account the recent drop of the Top mass from the Tevatron results. We are still within the $68 \%$ confidence level even in the event of a $1 \mathrm{TeV}$ composite Higgs mass.

[5] S. B. Gudnason, T. A. Ryttov, and F. Sannino, arXiv:hepph/0612230 [Phys. Rev. D (to be published)].

[6] K. Kainulainen, K. Tuominen, and J. Virkajarvi, Phys. Rev. D 75, 085003 (2007).

[7] C. Kouvaris, arXiv:hep-ph/0703266 [Phys. Rev. D (to be published)].

[8] S. B. Gudnason, C. Kouvaris, and F. Sannino, Phys. Rev. D 74, 095008 (2006)

[9] T. Appelquist, K. D. Lane, and U. Mahanta, Phys. Rev. Lett. 61, 1553 (1988).

[10] A. G. Cohen and H. Georgi, Nucl. Phys. B314, 7 (1989).

[11] T. Appelquist, J. Terning, and L. C. R. Wijewardhana, Phys. Rev. Lett. 77, 1214 (1996).
[12] V. A. Miransky and K. Yamawaki, Phys. Rev. D 55, 5051 (1997); 56, 3768 (1997).

[13] H. Gies and J. Jaeckel, Eur. Phys. J. C 46, 433 (2006).

[14] T. Banks and A. Zaks, Nucl. Phys. B196, 189 (1982).

[15] S. Weinberg, Phys. Rev. D 19, 1277 (1979); L. Susskind, Phys. Rev. D 20, 2619 (1979).

[16] E. Eichten and K. D. Lane, Phys. Lett. B 90, 125 (1980).

[17] B. Holdom, Phys. Rev. D 24, 1441 (1981).

[18] K. Yamawaki, M. Bando, and K. i. Matumoto, Phys. Rev. Lett. 56, 1335 (1986).

[19] T. W. Appelquist, D. Karabali, and L. C. R. Wijewardhana, Phys. Rev. Lett. 57, 957 (1986).

[20] K. D. Lane and E. Eichten, Phys. Lett. B 222, 274 (1989).

[21] N. Evans and F. Sannino, arXiv:hep-ph/0512080.

[22] S. Duane, A. Kennedy, B. Pendleton, and D. Roweth, Phys. Lett. B 195, 216 (1987).

[23] G. Divitiis, R. Frezzotti, M. Guagnelli, M. Masetti, and R. Petronzio, Phys. Lett. B 367, 279 (1996).

[24] B. Mawhinney, arXiv:hep-lat/9705030.

[25] Y. Iwasaki, K. Kanaya, S. Kaya, S. Sakai, and T. Yoshie, Phys. Rev. D 69, 014507 (2004).

[26] J. Kogut, H. Wyld, and D. Sinclair, Phys. Rev. Lett. 54, 1980 (1985) 